\title{
Compact Decoupled Antenna Pairs for 5G Applications
}

\author{
Mr. Arun. M \\ Assistant Professor (Sr. Grade) \\ Department of Electronics and Communication Engineering \\ Mepco Schlenk Engineering College \\ Virudhunagar, Sivakasi
}

\author{
Divya. C, Karthini. B \\ Department of Electronics and Communication Engineering \\ Mepco Schlenk Engineering College \\ Virudhunagar, Sivakasi
}

\begin{abstract}
In this letter, a compact self-decoupled antenna structure is proposed for the $5 \mathrm{G}$ multiple-input-multiple output (MIMO) operation in 5G tablets. The antenna structure consists of two adjacent antenna elements, which are placed very close to each other which is placed at a distance of $1.2 \mathrm{~mm}$ and located on the same side of the system ground plane. A compact selfdecoupled antenna pair can be obtained by sharing one common ground. The MIMO antenna system is optimized to operate in the 3.5 GHz (3.4 -3.6 GHz) band with isolation better than $35 \mathrm{~dB}$. An antenna prototype is fabricated, and good results for simulation and measurement is obtained. The gain obtained is 3.3dB. An antenna is fabricated and good results for simulation are obtained.
\end{abstract}

Index Terms - 5G, Tablet, Multi input multi output, Self decoupled, compact antenna.

\section{INTRODUCTION}

In the upcoming world, $5^{\text {th }}$ generation electronic devices will play a major role and it will have advantages such as higher transmission rate, high reliability, low latency when compared to $4 G[3]-[10]$. So the upcoming generation will mostly rely on $5 \mathrm{G}$ communication electronic devices. To meet the requirement of 5G, MIMO (multiple input multiple output) antennas operating in sub $6 \mathrm{GHz}$ band FR1 range $(410 \mathrm{MHz}-7.125 \mathrm{GHz})$ used in $5 \mathrm{G}$ electronic devices [1]-[3]. To meet high isolation in $5 \mathrm{G}$ electronic devices like $5 \mathrm{G}$ tablets becomes challenge in before years [4]. In past, many methods were proposed using neutralization line to improve isolation [2]-[5]. To overcome this problem, MIMO antenna structure is used.

In this letter, we have proposed four decoupled antennas shaped ring common ground to meet better isolation [6]-[8]. Four antennas split into two antenna pairs very close to each other. The antenna pairs shared a common ground and the antenna pairs are isolated by I shape isolating element [9]. The performance of our proposed antenna is verified using simulation in HFSS (high frequency structure simulator) and by fabrication.

\section{COMPACT DECOUPLED ANTENNA}

The dimensions of the compact decoupled antenna pairs are illustrated in the fig.1.1. The dimension of the substrate is $150 \mathrm{~mm} \times 75 \mathrm{~mm} \times 0.8 \mathrm{~mm}$ and the dimension of the ground plane

is $150 \mathrm{~mm} \times 73 \mathrm{~mm} \times 0.8 \mathrm{~mm}$. Two antenna frames are placed on the left and the right side of the substrate. On each of the antenna frame, antenna pairs are printed separately. The length of the antenna frame is $150 \mathrm{~mm}$ and the width is $0.8 \mathrm{~mm}$ and the height is $6.9 \mathrm{~mm}$. The system substrate and the antenna frames are based on the FR4 with relative permittivity (Er) 4.4 and dielectric loss tangent 0.02 . Each antenna pair consists of $U$ shaped radiating element, $U$ shaped feeding element and I shaped isolating element. U shape feeding element is used to obtain better gain and isolation loss. The length of the isolating element is $6.9 \mathrm{~mm}$ and the width is $0.8 \mathrm{~mm}$. The arrangement of the antenna pairs is done in such a way that below the $U$ shaped radiating element, $\mathrm{U}$ shape feeding element is placed in order to get good return loss. For better isolation an I shaped isolating element is place in between each antenna so that we can achieve good isolation loss. Microstrip feed is used to obtain better return loss. Here we use four microstrip feeds. Each micro- strip feed is fed with each feeding element directly. Length of each microstrip feed is $12.2 \mathrm{~mm}$. Two feeds are placed on the either side. Four microstrip feeds are fixed on the substrate. Each antenna pairs share a common ground. The horizontal coupling gap between $U$ shaped radiating element and $U$ shaped isolating element is $0.2 \mathrm{~mm}$. The gap between each radiating element is $1.2 \mathrm{~mm}$. Each antenna pairs are located in the middle of the antenna frame. All the antennas are fed with $50 \Omega$ SMA (sub miniature version A) connectors via hole from the back side of the system substrate .Four SMA connectors are used to connect the four microstrip feed with the ground.

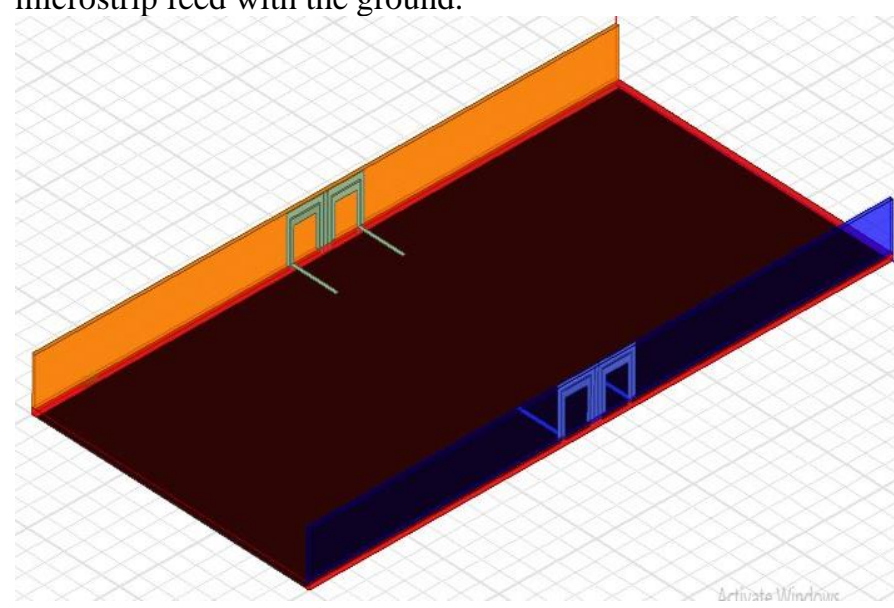

Fig 1.1.Antenna structure

\section{FORMULAS USED}

The wavelength is calculated using,

$$
\lambda=\frac{c}{f}
$$

c is the velocity of light and its value is $3 \times 10^{11} \mathrm{~mm}$ 
$\lambda$ is the wavelength

$\mathrm{f}$ is the frequency.

Width of the patch is given by,

$$
w=\frac{c}{2 f} \sqrt{\frac{2}{\varepsilon_{r}+1}}
$$

where $\varepsilon_{r}$ is the dielectric constant of the substrate .

Length of the patch is calculated by,

$$
L=L_{\text {eff }}-2 \Delta L
$$

where Leff is the effective length.

Effective length is given by,

$$
L_{\text {eff }}=\frac{c}{2 f \sqrt{\varepsilon_{r_{\text {eff }}}}}
$$

where greff is the effective dielectric constant

The normalized extension length is given by,

$$
\Delta L=0.412 h \frac{\left(\varepsilon_{\text {reff }}+0.3\right)\left(\frac{w}{h}+0.264\right)}{\left(\varepsilon_{\text {reff }}-0.258\right)\left(\frac{w}{h}+0.8\right)}
$$

where $h$ is the thickness of the substrate and $w$ is the width of the substrate.

Effective dielectric constant is given by,

$\varepsilon_{\text {reff }}=\frac{\varepsilon_{r+1}}{2}+\frac{\varepsilon_{r-1}}{2}\left[1+12 \frac{h}{w}\right]^{\frac{-1}{2}}$

where $\mathrm{h}$ and $\mathrm{w}$ are the thickness and width of the substrate respectively.

Length of the substrate is given by,

$$
L_{g=} L+6 h
$$

Width of the substrate is given by,

$$
w_{g}=w+6 h
$$

Height of the substrate is given by,

$$
h=\frac{0.0606 \lambda}{\sqrt{\varepsilon_{r}}}
$$

Guided wavelength is given by,

$$
\lambda_{g}=\frac{\lambda}{\sqrt{\varepsilon_{r_{\text {eff }}}}}
$$

The feed length is given by,

$$
L_{f}=\frac{\lambda_{g}}{4}
$$

\section{OBTAINTED RESULTS}

An antenna prototype of the proposed 4 antenna MIMO system was measured and fabricated. With the operating frequency of $3.5 \mathrm{GHz}$, the obtained return loss is about -35.8 $\mathrm{dB}$. The simulated result obtained is shown in the fig 1.2.

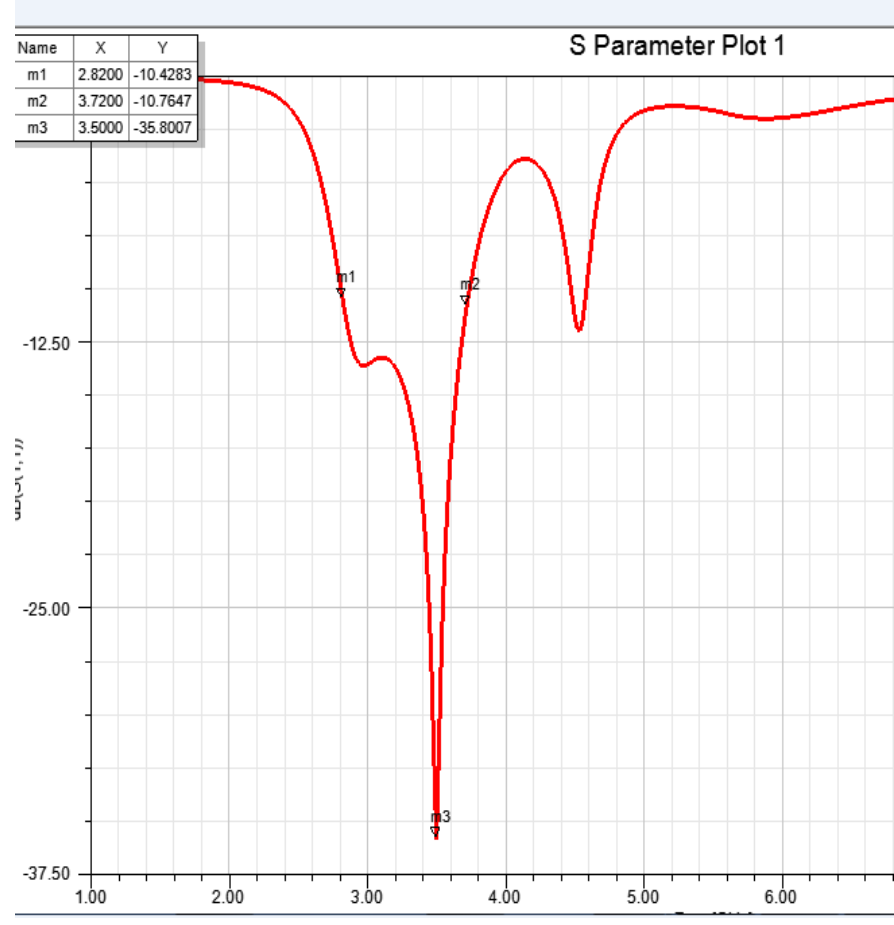

Fig 1.2 Return loss

The main purpose of the antenna is to get better isolation loss. The proposed antenna structure antenna has a better isolation loss of about $-32 \mathrm{~dB}$ and $-39.93 \mathrm{~dB}$. The isolation is obtained by the common grounding of the antenna pairs. The obtained result is shown in the figure 1.3 and fig 1.4. The radiation pattern obtained for the proposed antenna structure is omni directional and it is given in the fig 1.5 and a $3 \mathrm{D}$ plot is also obtained and it is shown in the fig 1.6 and the efficiency of the proposed antenna structure is $71 \%$ and a good arrangement for simulation and the measure is observed.

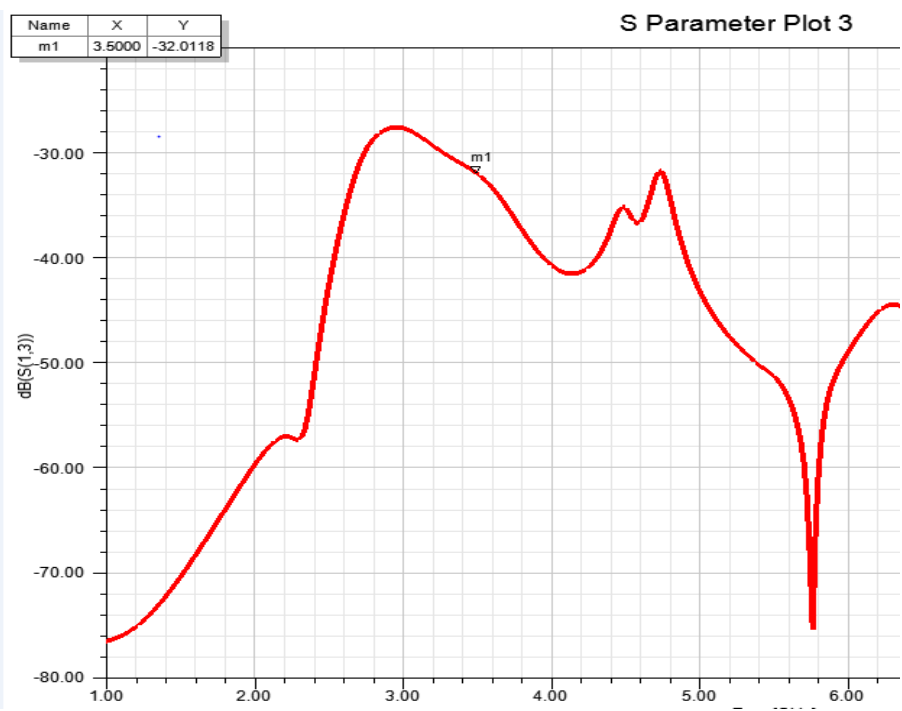

Fig1.3 Isolation loss 


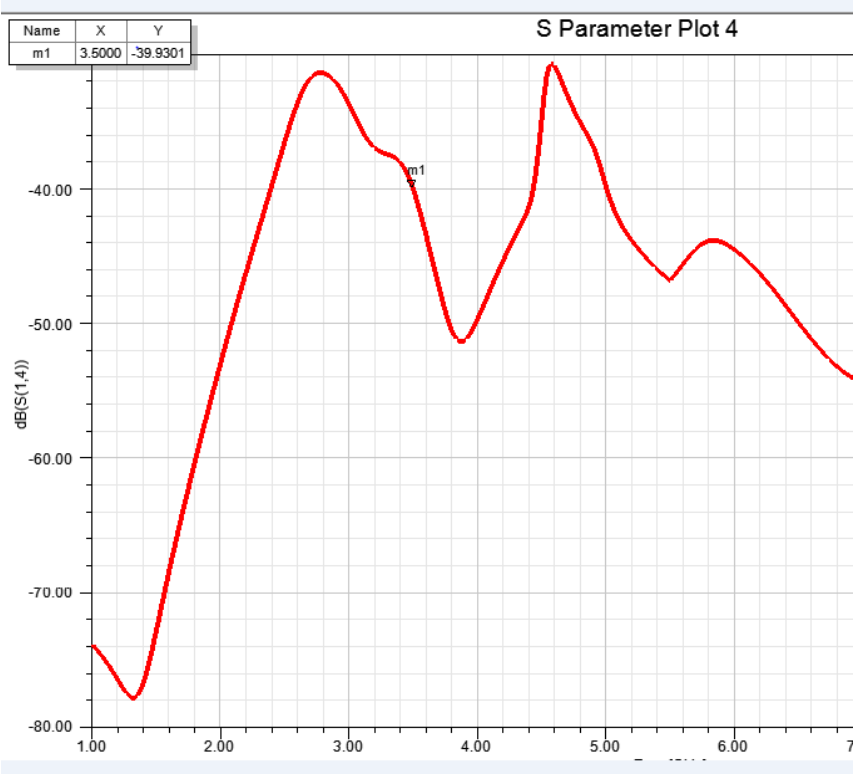

Fig 1.4.S parameter (S14)

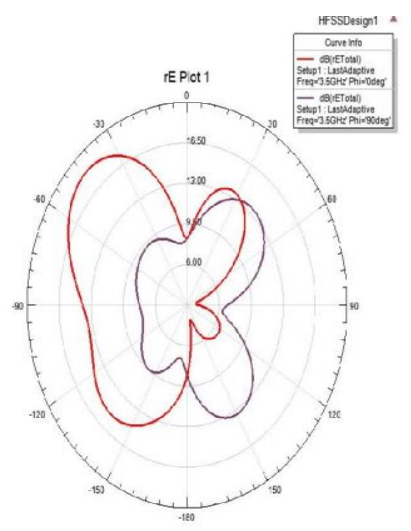

Fig 1.5 Radiation pattern of the proposed antenna structure
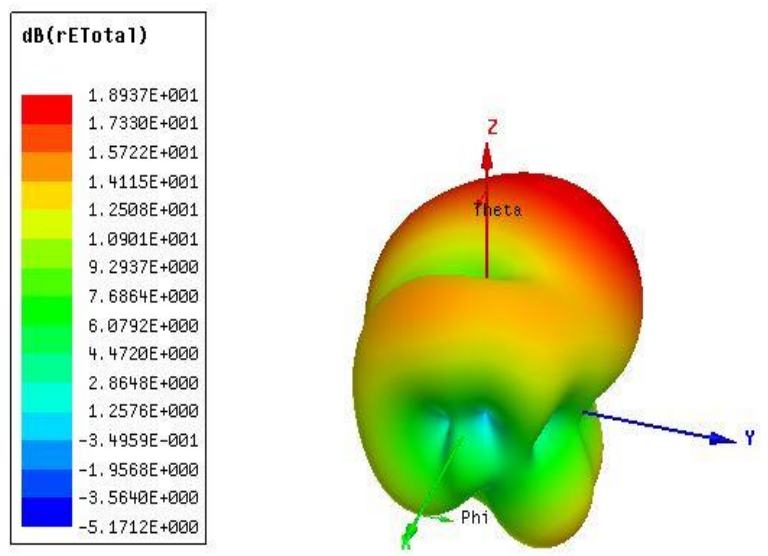

Fig 1.6 Simulated and measure 3D radiation pattern
The gain obtained for our proposed at $3.5 \mathrm{GHz}$ frequency is around $3.445 \mathrm{~dB}$ obtained in the fig 1.7 .

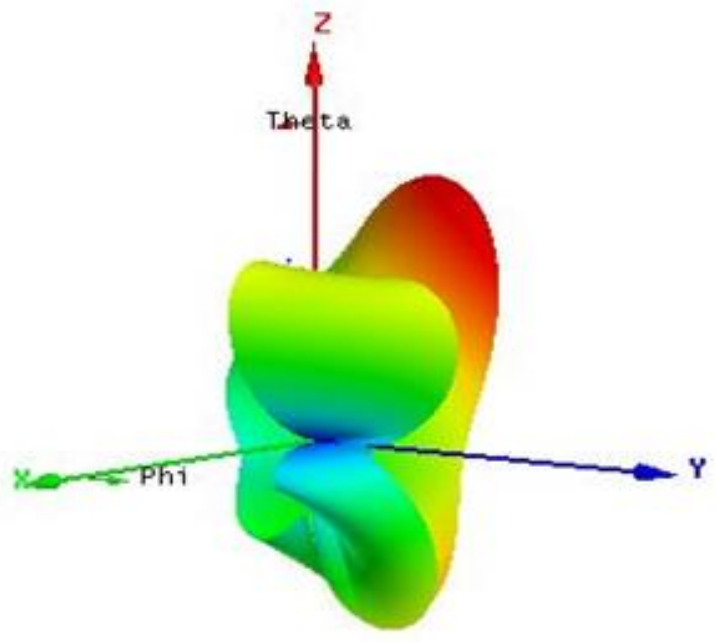

Fig 1.7 Gain obtained for the proposed compact decoupled antenna structure

The results obtained confirm that the performance of the MIMO antenna structure is more than enough for $5 \mathrm{G}$ applications.

\section{V.CONCLUSION}

A compact decoupled antenna pairs for $5 \mathrm{G}$ applications like $5 \mathrm{G}$ tablet was proposed. By having two antenna pairs and the antenna pairs share a common grounding, good isolation of about $-39 \mathrm{~dB}$ is achieved even there are very close to each other. The proposed antenna structure has good isolation and high and high return loss which is most suitable for the $5 \mathrm{G}$ applications. The efficiency obtained is also greater than $70 \%$ and it is more useful for $5 \mathrm{G}$ applications.

\section{ACKNOWLEDGMENT}

The authors thank the reviewers for valuable comments and the feedback.

\section{REFERENCES}

[1] L. Sun, H. Feng, Y. Li, and Z. Zhang, "Compact 5G MIMO mobile phone antennas with tightly arranged orthogonal- mode pairs," IEEE Trans. Antennas Propagation., vol. 66, no. 11, pp. 6364-6369, Nov. 2018

[2] Y. L. Ban, C. Li, C. Y. D. Sim, G. Wu, and K.L. Wong, "4G/5G Multiple Antennas for future Multi-mode Smartphone Applications,” IEEE Access, vol. 4, pp. 2981-2988, 2016.

[3] J. Y .Deng, J. Yao, D. Q. Sun, and L. X. Guo, "Ten-element MIMO antenna for 5G terminals," Microwave. Opt. Technol. Letter., vol. 60, pp. 2001-2010, Aug. 2018.

[4] K. L. Wong, B. W. Lin, and W. Y. Li, "Dual-band dual inverted F loop antennas as a compact decoupled building block for forming eight 3.5/5.8-GHz MIMO antennas in the future Smartphone," Microwave. Opt. Technol. Letter., vol. 59, pp. 2715-2721, Nov. 2017

[5] Y.WangandZ.Du, “A wide band printed dual antenna system with a novel neutralization line form obileterminals,"IEEE Antennas Wireless Propag. Lett., vol. 12, pp. 1428-1431, 2013

[6] A. Zhao and Z.Ren, "Size reduction of self-isolated antenna MIMO antenna system for $5 \mathrm{G}$ mobilephone applications,'IEEE Antennas Wireless Propag. Lett., vol. 18, pp. 152-156, 2019.

[7] K. L. Wong, J. Y. Lu, L. Y. Chen, W. Y. Li, and Y. L. Ban, "8antenna and 16-antenna arrays using the quad-antenna linear array as a building block for the $3.5 \mathrm{GHz}$ LTE MIMO operation in the 
Smartphone," Microwave Opt. Technology. Letter., vol. 58, pp. 174-181, Jan. 2016.

[8] K. L. Wong, Y. H. Chen, and W. Y. Li, "Decoupled compact ultrawideband MIMO antennas covering $3300-6000 \mathrm{MHz}$ for the fifthgeneration mobile and $5 \mathrm{GHz}$ WLAN operations in future smartphone,"Microw.Opt.Technol.Lett.,vol.60,pp.2345-2351,Oct.2 2018.

[9] M. Y. Li et al., "Eight-port orthogonally dual-polarized antenna array for 5G Smartphone applications," IEEE Transactions Antennas and Wave Propagation., vol. 64, no. 9, pp. 3820-3830, Sep. 2016

[10] J. G. Andrews et al., "What will 5G be?," IEEE J. Sel. Areas Communication., vol. 32, no. 6, pp. 1065-1082, Jun. 2014. 